\title{
Liquid-based Anal Cytology as a Screening Tool for Prevention of Anal Cancer in at-risk Populations: A Single-Center Retrospective Analysis on 111 Patients
}

\author{
Luigi Pisano $^{10}$ Luana Tiradritti ${ }^{1}$ Elisa Lorenzoni ${ }^{1}$ Giuliano Zuccati ${ }^{1}$ Prassede Foxi ${ }^{2}$ \\ Daniela Butera ${ }^{2}$ Marzia Matucci ${ }^{2}$ Massimo Confortini ${ }^{2}$ Francesca Carozzi ${ }^{2}$
}

${ }^{1}$ Department of Health Science, Section of Dermatology, University

Address for correspondence Luigi Pisano, MD, Department of Health of Florence, Florence, Italy

${ }^{2}$ Cancer Prevention and Research Unit, Florence, Italy Science, Section of Dermatology, University of Florence, Florence, Italy (e-mail: luigi.pisano88@yahoo.it).

J Coloproctol 2021;41(4):419-424.

Abstract

Keywords
- anal cancer
- anal cytology
- liquid-based cytology
- pap test
- screening

Objective Squamous cell carcinoma of the anus (SCCA) is associated with human papillomavirus (HPV) infection in almost $90 \%$ of the cases. Its incidence is alarmingly high among men who have sex with men (MSM) and continues to increase at an average rate of $2 \%$ per year. The objective of the present study is to evaluate the usefulness and performance of liquid-based anal cytology as a screening tool for prevention and early detection of SCCA in a cohort of at-risk men.

Method We conducted a retrospective study including $111 \mathrm{MSM}$, aged between 22 and 62 years old, who underwent anal cytological screening with a liquid-based Pap test at our sexually transmitted diseases (STDs) clinic from January 2015 to March 2017.

Results Out of 111 anal smears, $57(51,4 \%)$ resulted negative, $42(37,8 \%)$ abnormal, and $12(10,8 \%)$ unsatisfactory for the cytological evaluation. Only patients with an abnormal cytology underwent anoscopy and subsequent biopsy. The histological results were as follows: negative for squamous intraepithelial lesion (SIL) in 5 cases, low-grade SIL (L-SIL) in 21, high-grade SIL (H-SIL) in 5, SCCA in 1. Five patients had a normal anoscopy and biopsy was not taken.

Conclusion Liquid-based cytology, reducing the "darkening factors" typical for the conventional smears, has a higher positive predictive value than the traditional technique. Moreover, a cytological diagnosis of atypical squamous cells of undetermined significance (ASC-US) or L-SIL may hide a severe dysplasia or even a carcinoma. Thus, all patients with an abnormal anal cytology at any grade should be considered for anoscopy.

\section{Introduction}

Worldwide, as many as $4.5 \%$ of new cancer cases are associated with human papillomavirus (HPV) infection. ${ }^{1}$
Squamous cell carcinoma of the anus (SCCA) is the second most commonly HPV-related cancer, immediately after cervical cancer. Nearly $90 \%$ of anal cancers are attributable to HPV. ${ }^{2}$ received

December 31, 2020 accepted after revision June 15, 2021
DOI https://doi.org/

10.1055/s-0041-1736643. ISSN 2237-9363. (c) 2021. Sociedade Brasileira de Coloproctologia. All rights reserved.

This is an open access article published by Thieme under the terms of the Creative Commons Attribution-NonDerivative-NonCommercial-License, permitting copying and reproduction so long as the original work is given appropriate credit. Contents may not be used for commercial purposes, or adapted, remixed, transformed or built upon. (https://creativecommons.org/ licenses/by-nc-nd/4.0/)

Thieme Revinter Publicações Ltda., Rua do Matoso 170, Rio de Janeiro, RJ, CEP 20270-135, Brazil 
Based on data reported by the Surveillance, Epidemiology, and End Results Program (SEER), ${ }^{3}$ the number of new cases of anal cancer is 1.8 per 100,000 men and women per year. However, SCCA is one of the most frequent non-AIDS-defining neoplasms in HIV-positive patients, especially in HIV-positive men who have sex with men(MSM), with a very high incidence of up to 144 cases per 100,000 individuals per year. ${ }^{4-6}$ In the population of MSM, both HIV-positive and HIV-negative men, the incidence is alarmingly high and continues to increase at a gross rate of $2 \%$ with each passing year. ${ }^{7,8}$

In economically developed countries, cervical cancer in women has plummeted to a fifth of its initial incidence, as a result of cervical screening programs with the Pap smear. ${ }^{7}$ Based on the great success of cervical cytological screening, some authors ${ }^{9-13}$ have proposed a similar screening for the prevention of anal cancer in the at-risk male population. Cervical cancer and anal cancer share several similar characteristics: both cancers are strictly associated with a persistent infection of epithelial cells by oncogenic HPV types, and both are preceded by preinvasive neoplastic lesions (cervical intraepithelial neoplasia [CIN], in the cervix, anal intraepithelial neoplasia [AIN], in the anus), that may be identified with Pap smear. ${ }^{11-13}$

A two-tiered classification for intraepithelial lesions has been recently proposed by the Lower Anogenital Squamous Terminology Standardization (LAST) Project ${ }^{14}$ : low-grade squamous intraepithelial lesions (L-SIL) and high-grade squamous intraepithelial lesions (H-SIL). ${ }^{15,16}$ However, it has been found that anal condyloma, considered a variant of L-SIL, may also harbor foci of H-SIL or even invasive squamous cell carcinoma. The coexistence of different lesions in the same patient is due to the frequent detection of multiple HPVs, both high- and low-risk, in MSM, especially HIV-infected patients. ${ }^{17-20}$

A cytological screening with anal Pap test is usually performed in all MSM at the Sexually Transmitted Diseases (STDs) Center of the Dermatological Clinic of University of Florence. From 2013 to 2014, conventional anal Pap smears were performed, but since 2015 the STD Center of Florence has started to use liquid-based smears for anal cytology, in close analogy with what happened for cervical cytology.

We undertook a retrospective study on patients who underwent anal cytological screening with a liquid-based Pap test between 2015 and 2017. The aim of the present study was to evaluate the performance of liquid-based anal cytology in terms of adequacy and diagnostic accuracy. Moreover, our results were retrospectively compared with those obtained in a previous study ${ }^{9}$ from slides prepared by a conventional technique, to detect the best strategy in diagnosing anal intraepithelial neoplasia in HIV-infected and -uninfected MSM. A prospective study aimed to directly compare the two methods needs to be conducted in the near future.

\section{Method}

Data for the present retrospective study were collected at the STD Center of the Dermatological Clinic of University of Florence between January 2015 and March 2017.
All participants gave informed consent. After a clinical evaluation, they were subjected to an anal sampling for cytology. A nonsterile disposable cytobrush, ordinarily used for cervical Pap smear in females, was gently inserted between $\sim 3$ and $5 \mathrm{~cm}$ into the anal canal to obtain cells from the transformation zone. Once fully inserted, the endobrush was firmly rotated three times clockwise and three times anticlockwise, applying a vigorous lateral pressure on the walls of the anal canal, to maximize cellular yield. The brush was then removed, put in a liquid-based cytology fluid (ThinPrep) and rotated for $\sim 10$ seconds to facilitate cell detachment.

All samples were sent to the Cancer Prevention Laboratory of the Institute for the Study and Prevention of Cancer (ISPRO) of Florence. Liquid-based slides were prepared by the same processor used for cervicovaginal cytology. The processor mechanically disperses the cells, which are then drawn on a filter by negative pressure and transferred on a glass slide; at the end, the slides are stained with Papanicolaou stain, exactly as conventional smears. Each sample was examined simultaneously by four cytopathologists.

Several advantages of liquid-based cytology over conventional smears are documented, such as absence of obscuring material (blood, inflammatory cells, and mucus), lack of airdrying artifacts, and the presence of consistently well-preserved cells and a monolayer cellular surface that is easier to screen. ${ }^{21-23}$

Samples with scant cellularity or presence of numerous anucleated squamous cells were classified as "inadequate/inconclusive" for the cytological interpretation. The adequate cellularity of an anorectal sampling is similar to a cervical one: a slide with between 2,000 and 3,000 nucleated squamous cells is considered adequate. For thin-layer preparations, this is equivalent to 1 to 2 squamous nucleated cells/HPF for ThinPrep (diameter of $20 \mathrm{~mm}$ ).

The presence of rectal columnar cells and/or of metaplastic squamous cells is not a requirement for adequacy, but must still be reported, because it is an indication that the transformation zone has been sampled.

Anal cytological results were classified using the 2001 Bethesda System ${ }^{24}$ as: negative for intraepithelial lesion or malignancy (NILM), atypical squamous cells of undetermined significance (ASC-US), atypical squamous cells, cannot exclude high-grade squamous intraepithelial lesion (ASC-H), L-SIL, H-SIL, and carcinoma.

All patients who had an abnormal anal cytological result of ASC-US and above underwent anoscopy, always with application of $5 \%$ acetic acid solution, used to aid visualization of abnormal anal tissue. Anoscopy was performed with the patient in left lateral decubitus; an anoscope was placed into the anus with lidocaine lubrication, and then a gauze soaked in $5 \%$ acetic acid solution was inserted into the anal canal while the anoscope was gently removed. The gauze was removed after a few minutes to avoid a widespread "false positive" reaction. After application of the acetic acid solution, the anoscope was inserted again and the mucosa was carefully examined with the aid of a colposcope for lighting and magnification. When the anoscopy showed the presence of "aceto-white changes" characteristic of AIN, including flat 
Table 1 Liquid-based anal Pap test results based on HIV status

\begin{tabular}{|l|l|l|l|}
\hline & HIV-POSITIVE & HIV-NEGATIVE & TOTAL \\
\hline UNSATISFACTORY & 0 & $12(16 \%)$ & 12 \\
\hline NILM & $19(52.8 \%)$ & $38(50.7 \%)$ & 57 \\
\hline ASC-US & $6(16.7 \%)$ & $8(10.6 \%)$ & 14 \\
\hline ASC-H & 0 & $3(4 \%)$ & 3 \\
\hline L-SIL & $8(22.2 \%)$ & $12(16 \%)$ & 20 \\
\hline H-SIL & $3(8.3 \%)$ & $2(2.7 \%)$ & 5 \\
\hline $\begin{array}{l}\text { ANY ABNORMALITY } \\
\text { (ASC-US. ASC-H. L-SIL. H-SIL) }\end{array}$ & $17(47.2 \%)$ & $25(33.3 \%)$ & 42 \\
\hline TOTAL & $36(32.4 \%)$ & $75(67.6 \%)$ & 111 \\
\hline
\end{tabular}

Abbreviations: ASC-H, atypical squamous cells, which cannot exclude a high-grade lesion; ASC-US, atypical squamous cells of undetermined significance; H-SIL, high-grade squamous intraepithelial lesion; L-SIL, low-grade squamous intraepithelial lesion; NILM, negative for intraepithelial lesion or malignancy.

or slightly raised areas of thickened mucosa with or without vascular pattern abnormalities, a biopsy was performed and sent to the Pathologic Anatomy Department of Careggi for the definitive histopathological diagnosis.

\section{Results}

In the selected period, we screened for anal cancer 111 male patients aged between 22 and 62 years old (median age: 38 years old). All included patients were homo- or bisexual; 36 (32.4\%) were HIV-positive and 75 (67.6\%) were HIV-negative. At least 1 previous or concurrent sexually transmitted infection was found in $86(77.5 \%)$ patients: 62 (55.8\%) patients had a history of anogenital warts, $43(38.7 \%)$ patients had a history of syphilis, 35 (31.5\%) had a previous diagnosis of gonorrhea, 18 (16.2\%) had a previous anogenital infection by Chlamydia trachomatis, $12(10.8 \%)$ had a history of hepatitis B virus (HBV) infection, and 4 (3.6\%) had a concurrent hepatitis $\mathrm{C}$ virus (HCV) infection. All 36 HIV-positive patients included in the study were under treatment with Highly Active AntiRetroviral Therapy (HAART), with undetectable plasma viral load and a good immunological status.
Out of 111 anal Pap smears prepared using liquid-based slides (ThinPrep), 57 (51.4\%) resulted negative, 12 (10.8\%) unsatisfactory for the cytological evaluation, and 42 (37.8\%) abnormal.

Among abnormal anal Pap tests, 14 cases of ASCUS (12.6\%), 3 cases of ASC-H (2.7\%), 20 cases of LSIL (18\%), and 5 cases of HSIL (4.5\%) were identified.

Among the 111 participants included in the analyses, 36 (32.4\%) were HIV-infected MSM and 75 (67.6\%) were HIVuninfected MSM. In - Table 1, we report the anal Pap test results based on HIV status.

In our study, only participants with an abnormal anal cytological result from ASC-US and above had anoscopy, performed after application of $5 \%$ acetic acid solution.

The correlation between the cytological and histological results is shown in - Table 2 .

Among the 5 patients who had H-SIL as cytologic diagnosis, 2 patients (40\%) were lost to follow-up; in the 3 remaining patients, anoscopy always showed aceto-white lesions in the anal canal mucosa. Histological examination allowed to identify 2 cases of H-SIL ( 1 case of AIN II-III and 1 carcinoma in situ) and 1 case of infiltrating carcinoma.

Table 2 Correlation between anal cytology and histology results in 99 patients with adeguate cytology results

\begin{tabular}{|c|c|c|c|c|c|c|c|}
\hline \multirow[t]{2}{*}{ CYTOLOGY } & \multicolumn{7}{|c|}{ HISTOLOGY } \\
\hline & TOTAL & NO BIOPSY & NEGATIVE & INFLAMMATION & AIN I & AIN II-III, Cis & SCCA \\
\hline NILM & 57 & 57 & - & - & - & - & - \\
\hline ASC-US & 14 & 0 & 5 & 4 & 4 & 1 & 0 \\
\hline ASC-H & 3 & 0 & 0 & 0 & 2 & 1 & 0 \\
\hline L-SIL & 20 & $3^{*}$ & 0 & 1 & 15 & 1 & 0 \\
\hline $\mathrm{H}-\mathrm{SIL}$ & 5 & $2^{*}$ & 0 & 0 & 0 & 2 & 1 \\
\hline TOTAL & 99 & 62 & 5 & 5 & 21 & 5 & 1 \\
\hline
\end{tabular}

Abbreviations: AIN, anal intraepithelial neoplasia; ASC-H, atypical squamous cells, which cannot exclude a high-grade lesion; ASC-US, atypical squamous cells of undetermined significance; Cis, carcinoma in situ; H-SIL, high-grade squamous intraepithelial lesion; L-SIL, low-grade aquamous intraepithelial lesion; NILM, negative for intraepithelial lesion or malignancy; SCCA, squamous cell carcinoma of the anus.

*Patients who did not return to visit or refused to undergo anoscopy. 
Among the 20 patients with cytological report of L-SIL, 3 patients (15\%) refused to undergo anoscopy; in the other patients, anoscopy with application of $5 \%$ acetic acid solution and subsequent biopsy led to the histological diagnosis of LSIL (AIN I) in 15 (88.2\%) cases and of H-SIL (AIN II-III) in 1 (5.9\%) case. One patient received a diagnosis of infectious proctitis caused by the simultaneous presence of $\mathrm{C}$. trachomatis, Mycoplasma genitalium, Mycoplasma hominis and Ureaplasma urealyticum.

All 3 patients who had ASC-H on anal Pap test underwent anoscopy with biopsy: 2 (66.7\%) of them had a histological diagnosis of AIN I; only 1 (33.3\%) patient resulted in AIN II-III.

Among the 14 patients with cytological report of ASC-US, anoscopy was negative in 5 cases (35.7\%). In 4 patients (28.5\%), the histological examination identified a nonspecific inflammation: in 1 patient, the cytological examination also showed eggs of Enterobius vermicularis, for which the specific antihelminthic therapy was undertaken.

In the remaining 5 patients with ASC-US on Pap test, the anoscopy with subsequent biopsy and histological examination of the suspected aceto-white lesions led to the diagnosis of AIN I in 4 cases (28.6\%) and of AIN II-III in 1 case ( $7.1 \%$ of the total ASCUS).

The positive predictive value (PPV) of anal cytology was $64.3 \%$ in predicting histologic AIN I+ and $14.3 \%$ in predicting histologic AIN II +.

\section{Discussion}

Comparing data from HIV-infected and -uninfected homosexual males, our study revealed a significantly higher rate of anal cytological abnormalities in HIV-positive patients ( $47.2 \%$ versus $33.3 \% ; p=0,018$ ).

We found more L-SIL and H-SIL cases in HIV-positive MSM than in HIV-negative MSM $(p=0.04)$.

Comparing the results of the present study with data obtained from a previous cohort of patients subjected to conventional anal Pap smear, ${ }^{9}$ we observed a higher detection rate of anal cytological abnormalities with the use of liquid-based technique, probably due to the reduction of the "darkening factors" that were typical of conventional method: fecal and bacterial contamination, inflammation, and airdrying artifacts. ${ }^{21-23}$

While the detection rate of ASC-US and ASC-H did not undergo significant changes using the 2 different techniques, the number of L-SIL (18 versus $5.7 \%$ ) and H-SIL (4.5\% versus 0 ) cases showed a substantial increase. The difference between conventional and liquid-based anal cytology for detection of L-SIL and H-SIL was statistically significant $(p=0.022)$; in contrast, the difference was not statistically significant if all cytological abnormalities were considered $(p=0.19)$.

We found a rate of unsatisfactory samples of $10.8 \%$ using the liquid-based cytological technique, while in the previous study, ${ }^{9}$ anal Pap test was considered inconclusive/inadequate in $16.1 \%$ of the group tested with the conventional method.

Literature data did not show significant differences in sensitivity and specificity of cervical cytology to detect abnormal histologic diagnoses between conventional and liquid-based techniques. ${ }^{25}$ Studies of comparison between these two cytological techniques in anal cytology are more limited. $^{21-23,26}$

In our retrospective analysis, although limited by a small number of patients studied with various characteristics, the use of the liquid-based cytological technique has shown to be able to accurately read a greater number of anal cytological abnormalities compared with conventional Pap smears.

The PPV of anal cytology was $\sim 47.8 \%$ using a conventional slides preparation technique, as we had found 11 cases of AIN I+ among 23 abnormal Pap smears. ${ }^{9}$ Instead, using a liquidbased technique, the PPV rose up to $64.3 \%$ : in particular, we identified 27 cases of histological abnormalities AIN I+ among 42 abnormal Pap tests $(p=0.02)$.

Compared with the "end-point" of a histologically confirmed diagnosis of AIN II +, the PPV of anal cytology was $14.3 \%$ using liquid-based slides and $4.3 \%$ using conventional slides $^{9}(p=0.019)$.

From this indirect, retrospective comparison, we can deduce that liquid-based cytology, reducing the "darkening factors" typical of the conventional smears, is more diagnostic and allows us to have a higher PPV than the traditional technique. A prospective study that directly compares the two cytological techniques with the aim of confirming these observations needs to be conducted in the near future.

The greater diagnostic accuracy of liquid-based cytology can be also attributed to the different sampling technique: indeed, the cells obtained with this method, although in a smaller number, are not altered by rolling the swab directly on the glass slide, which is a traumatic and strongly operatordependent maneuver.

Out of the 6 cases who had a histologically-confirmed diagnosis of AIN II and above in our study, if referral to anoscopy was triggered by H-SIL alone, only 3 cases (50\%) would have been diagnosed on liquid-based cytology. This is in accordance with previous findings ${ }^{11,12,27}$ that showed that anal cytology was a poor predictor of the histologic grade of severity of lesions identified after biopsy.

Weiss et al. ${ }^{28}$ reported that even $67 \%$ of the cases of ASCUS corresponded to the presence of a high grade-AIN lesion on histological examination.

Compared with anal cytology, cervical cytology shows a greater degree of correlation with the histopathological findings ${ }^{29}$ : in fact, a cytological report of ASC-US or L-SIL hides the presence of a high-grade-CIN in only between 5 and $17 \%$ of cases. ${ }^{30}$ Instead, anal cytology has a high degree of correlation with histology only for the high-grade dysplasia (H-SIL), while there is a poor cytohistological agreement for the low-grade lesions (ASC-US / L-SIL). ${ }^{29}$

In our study in all three patients with H-SIL on Pap test who underwent anoscopy, we found a high-grade lesion (two cases of histologically confirmed AIN II-III/carcinoma in situ and one case of invasive carcinoma), but we also identified three cases of AIN II-III in patients with a cytological report of ASC-US, L-SIL and ASC-H, respectively.

Our findings are in accordance with literature data, ${ }^{9,11,12,17-19}$ also confirming that a cytological diagnosis of low-grade lesion, such as L-SIL or ASC-US, may effectively 
hide a severe dysplasia or even a carcinoma, which can be confirmed only with an accurate anoscopy and subsequent biopsy for histological examination. This suggests that all patients with an abnormal screening anal cytological result at any grade should be considered for anoscopy, preferably high-resolution anoscopy (HRA). ${ }^{26-29}$

\section{Conclusions}

Anal cytology as a screening tool for the detection of HPVrelated precancerous lesions and anal cancer is becoming more useful, especially in "high-risk" groups, such as HIVpositive patients and MSM.

Many factors, including air-drying and mechanical artifacts, bacteria, fecal material, inflammation, and scant cellularity, may compromise the adequacy of anal cytological smears prepared with the conventional technique. Liquidbased cytology, reducing these obscuring factors, allows us to obtain more satisfactory cytological samples with a higher number of diagnoses of SILs. Therefore, the use of the liquidbased technique may dramatically increase the utility of anal cytological screening.

However, even in well-prepared liquid-based slides, anal cytology continues to show a poor degree of correlation with histological findings; so, all patients with an abnormal anal Pap test, ASC-US or worse, should undergo anoscopy (preferably HRA) and eventually biopsy, which remains the "gold standard" for the diagnosis of AIN.

The lack of HRA investigation results in patients with negative cytology represents an important limitation of our work. A larger prospective study directly comparing liquidbased cytology with the conventional method and involving HRA and biopsy for all subjects undergoing anal cytological screening is necessary.

\section{Conflict of Interests}

The authors have no conflict of interests to declare.

\section{References}

1 de Martel C, Plummer M, Vignat J, Franceschi S. Worldwide burden of cancer attributable to HPV by site, country and HPV type. Int J Cancer 2017;141(04):664-670. Doi: 10.1002/ ijc.30716

2 Viens LJ, Henley SJ, Watson M, et al. Human PapillomavirusAssociated Cancers - United States, 2008-2012. MMWR Morb Mortal Wkly Rep 2016;65(26):661-666. Doi: 10.15585/mmwr. mm6526a1

3 Cancer Stat Facts. Anal Cancer (National Cancer Institute Web site). Accessed March 18, 2018 at: https://seer.cancer.gov/statfacts/anus. html

4 Hidalgo-Tenorio C, Gil-Anguita C, Ramírez-Taboada J, et al. Risk factors for infection by oncogenic human papillomaviruses in HIV-positive MSM patients in the ART era (2010-2016). Medicine (Baltimore) 2017;96(39):e8109. Doi: 10.1097/MD.00000000 00008109

5 Silverberg MJ, Lau B, Justice AC, et al; North American AIDS Cohort Collaboration on Research and Design (NA-ACCORD) of IeDEA. Risk of anal cancer in HIV-infected and HIV-uninfected individuals in North America. Clin Infect Dis 2012;54(07):1026-1034. Doi: $10.1093 /$ cid/cir1012
6 Powles T, Robinson D, Stebbing J, et al. Highly active antiretroviral therapy and the incidence of non-AIDS-defining cancers in people with HIV infection. J Clin Oncol 2009;27(06):884-890. Doi: 10.1200/JCO.2008.19.6626

7 Leeds IL, Fang SH. Anal cancer and intraepithelial neoplasia screening: A review. World J Gastrointest Surg 2016;8(01): 41-51. Doi: $10.4240 /$ wjgs.v8.i1.41

8 Nelson RA, Levine AM, Bernstein L, Smith DD, Lai LL. Changing patterns of anal canal carcinoma in the United States. J Clin Oncol 2013;31(12):1569-1575. Doi: 10.1200/JCO.2012.45.2524

9 Pisano L, Tiradritti L, Lorenzoni E, et al. Pap smear in the prevention of HPV-related anal cancer: preliminary results of the study in a male population at risk. G Ital Dermatol Venereol 2016;151 (06):619-627

10 Glynne-Jones R, Nilsson PJ, Aschele C, et al. Anal cancer: ESMOESSO-ESTRO Clinical Practice Guidelines for diagnosis, treatment and follow-up. Ann Oncol 2014 Sep;25 Suppl 3:iii10-20

11 Darragh TM, Winkler B. Anal cancer and cervical cancer screening: key differences. Cancer Cytopathol 2011;119(01):5-19. Doi: 10.1002/cncy.20126

12 Liszewski W, Ananth AT, Ploch LE, Rogers NE. Anal Pap smears and anal cancer: what dermatologists should know. J Am Acad Dermatol 2014;71(05):985-992. Doi: 10.1016/j.jaad.2014.06.045

13 Jin F, Grulich AE, Poynten IM, et al; SPANC Study Team. The performance of anal cytology as a screening test for anal HSILs in homosexual men. Cancer Cytopathol 2016;124(06):415-424. Doi: $10.1002 /$ cncy.21702

14 Darragh TM, Colgan TJ, Cox JT, et al; Members of LAST Project Work Groups. The Lower Anogenital Squamous Terminology Standardization Project for HPV-Associated Lesions: background and consensus recommendations from the College of American Pathologists and the American Society for Colposcopy and Cervical Pathology. Arch Pathol Lab Med 2012;136(10):1266-1297. Doi: 10.5858 /arpa.LGT200570

15 Clavero O, McCloskey J, Molina VM, et al. Squamous intraepithelial lesions of the anal squamocolumnar junction: Histopathological classification and HPV genotyping. Papillomavirus Res 2017; 3:11-17. Doi: 10.1016/j.pvr.2016.12.001

16 Doorbar J. Papillomavirus life cycle organization and biomarker selection. Dis Markers 2007;23(04):297-313. Doi: 10.1155/2007/ 613150

17 Schlecht HP, Fugelso DK, Murphy RK, et al. Frequency of occult high-grade squamous intraepithelial neoplasia and invasive cancer within anal condylomata in men who have sex with men. Clin Infect Dis 2010;51(01):107-110. Doi: 10.1086/653426

18 Pimenoff VN, Félez-Sánchez M, Tous S, et al. Disagreement in high-grade/low-grade intraepithelial neoplasia and high-risk/low-risk HPV infection: clinical implications for anal cancer precursor lesions in HIV-positive and HIV-negative MSM. Clin Microbiol Infect 2015;21(06):605.e11-605.e19. Doi: 10.1016/j. cmi.2015.02.009

19 McCloskey JC, Metcalf C, French MA, Flexman JP, Burke V, Beilin LJ. The frequency of high-grade intraepithelial neoplasia in anal/perianal warts is higher than previously recognized. Int J STD AIDS 2007;18(08):538-542

20 Richel O, Quint KD, Lindeman J, et al. One lesion, one virus: individual components of high-grade anal intraepithelial neoplasia in HIV-positive men contain a single HPV type. J Infect Dis 2014;210(01):111-120. Doi: 10.1093/infdis/jiu052

21 Darragh TM, Jay N, Tupkelewicz BA, Hogeboom CJ, Holly EA, Palefsky JM. Comparison of conventional cytologic smears and ThinPrep preparations from the anal canal. Acta Cytol 1997;41 (04):1167-1170. Doi: 10.1159/000332840

22 Sherman ME, Friedman HB, Busseniers AE, Kelly WF, Carner TC, Saah AJ. Cytologic diagnosis of anal intraepithelial neoplasia using smears and cytyc thin-preps. Mod Pathol 1995;8(03):270-274

23 Phanuphak N, Teeratakulpisarn N, Lim C, et al. Comparable performance of conventional and liquid-based cytology in 
424 Liquid-based Anal Cytology as a Screening Tool for Prevention of Anal Cancer Pisano et al.

diagnosing anal intraepithelial neoplasia in HIV-infected and -uninfected Thai men who have sex with men. J Acquir Immune Defic Syndr 2013;63(04):464-471

24 Solomon D, Davey D, Kurman R, et al; Forum Group Members Bethesda 2001 Workshop. The 2001 Bethesda System: terminology for reporting results of cervical cytology. JAMA 2002;287(16):2114-2119. Doi: 10.1001/jama.287. 16.2114

25 Ronco G, Confortini M, Maccalini V, et al. Uso della citologia in fase liquida nello screening dei precursori del cancro del collo dell'utero. Epidemiol Prev 2012;5(S2):e1-33

26 Chiao EY, Giordano TP, Palefsky JM, Tyring S, El Serag H. Screening HIV-infected individuals for anal cancer precursor lesions: a systematic review. Clin Infect Dis 2006;43(02):223-233. Doi: $10.1086 / 505219$

27 Arain S, Walts AE, Thomas P, Bose S. The Anal Pap Smear: Cytomorphology of squamous intraepithelial lesions. Cytojournal 2005;2(01):4. Doi: 10.1186/1742-6413-2-4
28 Weis SE, Vecino I, Pogoda JM, et al. Prevalence of anal intraepithelial neoplasia defined by anal cytology screening and highresolution anoscopy in a primary care population of HIV-infected men and women. Dis Colon Rectum 2011;54(04):433-441. Doi: 10.1007/DCR.0b013e318207039a

29 Repiso Jiménez JB, Frieyro-Elicegui M, Padilla-España L, PalmaCarazo F, de la Torre Lima J, Rivas-Ruiz F. Anal intraepithelial neoplasia in a sexually transmitted diseases outpatient clinic: correlation with cytological screening. J Eur Acad Dermatol Venereol 2014;28(05):658-661. Doi: 10.1111/jdv.12109

30 ASCUS-LSIL Traige Study (ALTS) Group. A randomized trial on the management of low-grade squamous intraepithelial lesion cytology interpretations. Am J Obstet Gynecol 2003;188(06):1393-1400. Doi: $10.1067 / \mathrm{mob} .2003 .462$ 